\section{The IUPAP Guidelines}

1 The selection of experiments and the priority accorded to them are the responsibility of the host facility.

2 The criteria to be used in selecting experiments and determining their priority are: scientific merit; technical feasibility; capability of the experimental group; availability of the resources required.

3 The institutional, regional or national affiliations of the experimental teams should not influence the selection of an experiment nor the priority accorded to it.

4 In some instances, no additional

\section{Major Facilities Defined}

By a major user facility, IUPAP means a regional, national or international facility with unique experimental capabilities of sufficient power and utility to attract a large number of visiting users, usually of the order of hundred or capabilities are needed to successfully perform an approved experiment other than those capabilities routinely provided by the host facility. When this is not the case, the contributions of each team and of the host facility are to be agreed upon by the authorized leaders of the team and by the host facility prior to approval. Whenever appropriate, these agreements should be formally drawn, and be accompanied by discussions with the relevant authorities for the regions concerned.

5 Host facilities should not normally require experimental groups to contribute to the running costs of the facilities (including associated experimental areas and equipment normally maintained by the facilities). Exceptions to this Guideline may be made in special circumstances or in the case of proprietary research, which we define as research the results which are not intended for timely disclosure in the open scientific literature. Such circumstances will not be invoked as an excuse to restrict access artificially nor exclude participation. Should a user facility feel that special circumstances exist, the facility should make public their reasons for a deviation from the normal procedure and the conditions for access by scientific groups. In all cases, special consideration should be given to the needs of less-developed nations. even thousands. Most high-energy and nuclear particle accelerator centres are in this category, as are plasma confinement facilities. To these we add accelerator facilities designed to produce and use synchrotron radiation, spallation neutron sources and nuclear reactors designed to produce neutrons for research purposes. Other, but by no means exhaustive, examples may include centres for high-resolution electron microscopy and high pressure or high-magnetic field research. The pattern of use at some of these facilities involves large teams of users.

\section{Roundup}

Neutron Demand Increasing

A panel discussion led by $\mathrm{H}$ Curien that followed summaries by 10 working groups at the workshop Scientific Prospects for Neutron Scattering with Present and Future Sources (Autrans; 11-13 January 1993 - organized by the European Science Foundation in collaboration with the European Neutron Scattering Association) voiced two main concerns. First, that demand for beam time at the already oversubscribed large sources will continue to build up as many smaller reactor neutron sources built in the 1960 s are closing down. Second, that a major new source such as the proposed European Spallation Source will be expensive, take a long time to design and build, and need meticulous justification. Demand is also being accentuated by the fact that most areas see a strong trend from "simple samples" towards complex systems. Moreover, scientists are tending to carry out systematic, time-consuming studies. There was general agreement that the techniques offered at the rapidly evolving third-generation synchrotron $\mathrm{x}$-ray sources are truly complementary. Nonetheless, a shortage of neutron beam time seems likely, and there remain pressing scientific and technological problems which call for the unique characteristics of neutrons combined with higher fluxes and more sophisticated instruments.

\section{ELFE at DESY}

NuPECC, the European Nuclear Physics European Coordination Committee, has established an Initiative Group for ELFE at DESY, Hamburg, to explore the common interface between ELFE (Electron Accelerator for Europe) and DESY's proposed TESLA linear collider and its project to build a high-power free-electron laser [see EN 26 (1995) 127]. NuPECC has proposed ELFE as Europe's "natural and necessary machine for the long-term future of hadronic physics", but there remains the need to assemble a large enough community with the necessary resources.

\section{GSI to Examine Upgrade}

The Gesellschaft für Schweri onenforschung (GSI), Darmstadt, has formed working groups to analyse in detail within one year a next-generation upgrade of its facilities. GSI produced its first beams with the UNILAC linear accelerator in 1975 and an upgrade that ended in 1990 involved installing the SIS heavy-ion synchrotron and the ESR storage ring. The topics selected are:

- deep-inelastic electron-nucleon scattering and electron-nucleus scattering at intermediate energies, taking account of projects such as the lower energy ELFE and DESY's higher energy HERA;

- $\mathrm{x}$-ray spectroscopy and radiation physics, notably high-brilliance $x$ rays as a by-product of high-quality electron beams;

- nuclear collisions at maximum baryon density (a high-energy synchrotron or a high-energy heavy-ion collider);

- physics with secondary beams from a high-energy synchrotron; - nuclear structure at an advanced radioactive-beam facility;

- plasma physics with heavy-ions to extend today's density and temperature limits.

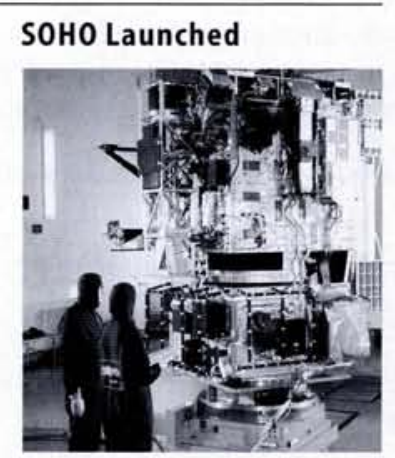

$\mathrm{SOHO}$ 's complex instrumentation was evident during assembly.

ESA's 1.6 tonne SOHO (Solar and Heliospheric Observatory) spacecraft was launched and deployed by NASA on 2 December 1995. Aboard are eight instruments provided by Europe and three by the US. SOHO is part of the international Solar-Terrestrial Science Programme, the next member of which is Cluster, a flotilla of four spacecraft to study how the Sun affects Earth and surrounding space that will be launched aboard ESA's new Ariane 5 in May. SOHO will take four months to reach its final position, the Lagrange point some 1.5 million $\mathrm{km}$ from Earth where the gravitational pull of the Earth and Sun are equal. With an unobstructed view, $\mathrm{SOHO}$ will then allow scientists for the first time to study 24 hours a day, 365 days a year, the internal structure of the Sun, its outer atmosphere and the origin of the solar wind. The first experimenter command was uplinked on 4 December and on 5 December, the VIRGO instrument made the first measurement when a cover was briefly opened to prove that it was properly released. Subsystems and instruments will be checked out during the fourmonth transfer phase.

Particle Hits Affect ISO

ESA's 2.4-tonne/o.6-m aperture Infrared Space Observatory (ISO), the last telescope of its type to be launched this century, was successfully placed in an eccentric, 24-hour orbit about Earth by ESA on 16 November 1995. The helium cryostat's cover was ejected on 27 November thereby enabling astronomical use. Data indicate that the absolute pointing error is better than specification. But all four instruments are affected by high rates (typically 1 hit/s) of cosmicparticle hits. The hits primarily increase noise, but have sometimes led to changes in instrument settings and observing strategy. Since ISO has an 18-month expected operational lifetime, the good news concerns tests indicating that a 40minute extension of observation time may be possible, for the present orbit, during the 16 hours/day spent outside the van Allen belts. 